\section{Suspected Airborne Transmission of MRSA on Surgical Ward}

Shiomori and coinvestigators, from the University of Occupational and Environmental Health, School of Medicine, Kitakyushu, Japan, conducted a study to investigate quantitatively the existence of airborne methicillinresistant Staphylococcus aureus (MRSA) in a hospital environment and to perform phenotyping and genotyping of MRSA isolates to study MRSA epidemiology. They performed prospective surveillance of patients with MRSA infections or colonization. Air samples were taken by an air sampler, and samples were obtained from object surfaces. An epidemiological study of MRSA isolates was performed with antibiotic susceptibility tests, coagulase typing, and pulsed-field gel electrophoresis. The study was conducted in three single-patient rooms in a 37-bed otolaryngology head-and-neck surgery unit. Three patients with squamous cell head-and-neck cancer were observed to have been colonized or infected with MRSA after surgery.

The MRSA samples were collected from the air in single-patient rooms during both a period of rest and when bed sheets were being changed. Isolates of MRSA were detected in all stages of the air sampler (from stage 1 [ $>7$ $\mu \mathrm{m}]$ to stage $6[0.65-1.1 \mu \mathrm{m}])$. Approximately $20 \%$ of the MRSA particles were within a respirable range of less than $4 \mu \mathrm{m}$. MRSA also was isolated from inanimate environments, such as sinks, floors, and bed sheets, in the rooms of the patients with MRSA infections, as well as from the patients' hands. An epidemiological study demonstrated that clinical isolates of MRSA in a patient ward were of one origin and that the isolates from the air and from inanimate environments were identical to the MRSA strains that caused infection or colonization in the inpatients.

The authors concluded that MRSA was recirculated among the patients, the air, and the inanimate environments, especially when there was movement in the rooms. Airborne MRSA may play a role in MRSA colonization in the nasal cavity or in respiratory tract MRSA infections. Measures should be taken to prevent the spread of airborne MRSA to control nosocomial MRSA infection in hospitals.

FROM: Shiomori T, Miyamoto $H$, Makishima K. Significance of airborne transmission of methicillinresistant Staphylococcus aureus in an otolaryngology-head and neck surgery unit. Arch Otolaryngol Head Neck Surg 2001;127:644-648.

\section{Nosocomial Infection Surveillance in US Children's Hospitals}

Nosocomial infections (NIs) and antimicrobial resistance are major causes of mortality and morbidity and have become a major public health focus. To date, most national and international NI surveillance and prevention activities have been focused on adults, despite the fact that pediatric patients are at high risk for NIs because of their immature immune systems and prevalent device use. In 1997 the
Hospital Infections Program at the CDC and the National Association of Children's Hospitals and Related Institutions partnered to establish a Pediatric Prevention Network. Infection control professionals and their hospital administrators at all children's hospitals were invited to participate. The objectives of the network are to establish baseline infection rates; design, implement, and evaluate prevention interventions; establish benchmark rates and best practices; and serve as a site for multicenter studies to improve outcomes for hospitalized children. This network serves as a model for quality improvement systems in health care.

Fifty participating children's hospitals were surveyed in 1998 to determine NI surveillance methods used and neonatal intensive care unit (NICU) and pediatric intensive care unit (PICU) 1997 NI rates. Data were collected on standardized forms and entered and analyzed by using SPSS for Windows (SPSS, Chicago, IL).

Forty-three (86\%) children's hospitals returned a completed questionnaire. All reported conducting NICU and PICU NI surveillance (range, 2-12; median, 12 months). Nineteen children's hospitals provided NICU NI rate data in one or more formats suitable for comparison. Denominators used for NICU NI rate calculations varied: 17 reported overall NI by patient-days; 19 reported bloodstream infection (BSI) by central venous catheter (CVC)days, and 8 reported BSI by patient-days. Sixteen children's hospitals reported NICU BSI data stratified by CVC-days and birth-weight cohort, and ventilator-associated pneumonia (VAP) by birth-weight cohort was reported by 12 . Twenty-four children's hospitals reported PICU NI rate data in one or more formats suitable for comparison. Denominators used for PICU NI rate calculations also varied: 20 reported overall NI rates by patient-days; 23 reported BSI rates by CVC-days, and 10 reported BSI rates by patient-days; 24 reported VAP by ventilator-days; and 15 reported urinary tract infections (UTIs) by urinary catheter-days. Median overall NI rates per 1,000 patientdays were 8.9 in NICUs and 13.9 in PICUs. Median NICU NI device-associated rates by birth weight $(>2,500 \mathrm{~g}, 1,501$ $2,500 \mathrm{~g}, 1,001-1,500 \mathrm{~g}$, and $\leqslant 1,000 \mathrm{~g}$ ) were as follows: BSI: $4.4,4.7,8.9$, and 12.6 ; and VAP: $0.9,1.1,4.9$, and 3.5 , respectively. Median PICU NI rates per 1,000 device-days were 6.5 for BSI; 3.7 for VAP; and 5.4 for UTI.

It was concluded that the number of months that NICU or PICU NI surveillance was conducted varied among hospitals. Reported NICU and PICU NI rates varied by hospital; some reported overall NI rates, and others focused on one or more particular sites of infection (eg, BSI or pneumonia). Many did not provide NICU deviceassociated rates stratified by birth-weight group. Denominators used to calculate device-associated infection rates also varied, with hospitals reporting either patientdays or device-days. These findings suggest the need to determine reasons for variations and to identify optimal NI surveillance methods at children's hospitals, so that valid interhospital NI rate comparisons can be made.

FROM: Stover BH, Shulman ST, Bratcher DF, Brady MT, Levine GL, Jarvis WR. Pediatric Prevention Network nosocomial infection rates in US children's hospitals' 
neonatal and pediatric intensive care units. Am J Infect Control 2001;29:152-157.

Girouard S, Levine G, Goodrich K, Jones S, Keyserling H, Rathore M, et al. Pediatric Prevention Network: a multicenter collaboration to improve health care outcomes. Am J Infect Control 2001;29:158-161.

\section{Antibiotic-Resistant Bacteria Among Long-Term-Care Facility Residents}

There is limited information on antibiotic-resistant bacteria in community long-term-care facilities (LTCFs). Mylotte and coinvestigators have reported on a study whose objective was to obtain data on resistant organisms in residents from community LTCFs admitted to an inpatient acute geriatrics service (AGS). Two studies were performed. In the first study, bacteriology records of all admissions to the AGS for the period from November 1, 1998 , through June 30,2000 , were reviewed for resistant organisms (methicillin-resistant Staphylococcus aureus [MRSA], vancomycin-resistant enterococci [VRE], and resistant gram-negative bacilli). In the second study, residents admitted to the AGS during a 2-month period ( $\mathrm{n}=92$ admissions) had surveillance cultures (nares, gastrostomy site, wounds, and urine) for resistant organisms performed within 72 hours of admission.

In the retrospective study, there were 727 admissions, of which $437(60 \%)$ had 928 cultures within 72 hours of admission; $590(64 \%)$ cultures grew one or more pathogens. Urine (65\%) and blood (26\%) cultures accounted for $91 \%$ of all cultures done. Rates of resistance by culture site were as follows: urine (resistant organism in 16.6\% of 373 cultures), blood ( $6.7 \%$ of 60 cultures), wound (52\% of 23 cultures), and sputum ( $40 \%$ of 20 cultures). MRSA and enterococci with high-level gentamicin resistance were the most common resistant organisms identified. No VRE were isolated; only $3 \%$ of $421 \mathrm{gram}$-negative isolates were considered resistant strains, compared with $19 \%(P<.001)$ of grampositive isolates. In the prospective study, $17 \%$ of 92 residents were found to have a resistant organism in 1 or more surveillance cultures; the most common resistant organisms were MRSA and high-level gentamicin-resistant enterococci. Only 1 resident was found to have VRE in a rectal swab culture; resistant gram-negative bacilli also were uncommon.

The authors concluded that, among residents of community LTCFs admitted to an AGS, resistant organisms were identified infrequently ( $<20 \%$ of admissions). MRSA was the most common resistant organism; VRE and resistant gram-negative bacilli were rare. These findings vary from other studies suggesting that there may be geographic variation in the epidemiology of resistant organisms among residents of community LTCFs.

FROM: Mylotte JM, Goodnough S, Tayara A. Antibiotic-resistant organisms among long-term care facility residents on admission to an inpatient geriatrics unit: retrospective and prospective surveillance. Am J Infect Control 2001;29:139-144.

\section{MRSA in Latin America: 1996 to 1998}

Aires De Sousa and colleagues from the Universidade Nova de Lisboa, Oeiras, Portugal, studied 493 methicillinresistant Staphylococcus aureus (MRSA) isolates recovered from 1996 to 1998 from 22 hospitals in five countries of Latin America: Argentina, Brazil, Chile, Uruguay, and Mexico. The isolates were examined for antimicrobial susceptibility and clonal type so as to define the endemic clones in those hospitals. The hybridization of $\mathrm{ClaI}$ restriction digests with the mecA- and Tn554-specific DNA probes combined with pulsed-field gel electrophoresis of chromosomal SmaI digests (ClaI-mecA::ClaI-Tn554::PFGE clonal types) documented not only the predominance and persistence of the Brazilian clone (XI::B::B) in Brazil (97\%) and Argentina $(86 \%)$ but also its massive dissemination to Uruguay (100\%). Moreover, a close relative of the Brazilian clone (XI::kappa::B) was highly represented in Chile (53\%), together with a novel clone (47\%) (II:: $\left.\mathrm{E}^{\prime}:: \mathrm{F}\right)$ resistant to penicillin, oxacillin, ciprofloxacin, chloramphenicol, clindamycin, erythromycin, and gentamicin. A unique clonal type (I::NH::M) was detected in Mexico among pediatric isolates and was resistant to penicillin, oxacillin, and gentamicin only.

This study clearly documented the very large capacity for geographic expansion and the persistence of the Brazilian clone, contributing not only to the increasing uniformity of the MRSA in South America but worldwide as well.

FROM: Aires De Sousa M, Miragaia M, Santos Sanches I, Avila S, Adamson I, Casagrande ST, et al. Threeyear assessment of methicillin-resistant Staphylococcus aureus clones in Latin America from 1996 to 1998. J Clin Microbiol 2001;39:2197-2205.

\section{Povidone-Iodine Versus Chlorhexidine Gluconate-Impregnated Dressing for Prevention of CVC Infections in Neonates}

Neonates who require a central venous catheter (CVC) for prolonged vascular access experience high rates of catheter-related (CR) bloodstream infection (BSI). Garland and coinvestigators conducted a multicenter, randomized clinical trial to ascertain the efficacy of a novel chlorhexidine-impregnated dressing (Biopatch Antimicrobial Dressing; Johnson \& Johnson Medical, Arlington, TX) on the CVC sites of neonates for the prevention of catheter-tip colonization, CR BSI, and BSI without a source. The trial was conducted in six level III neonatal intensive care units. Patients studied were neonates admitted to study units who would require a CVC for at least 48 hours. Eligible infants were randomized before catheter placement to 1 of the 2 catheter-site antisepsis regimens: (1) $10 \%$ povidone-iodine (PI) skin scrub, or (2) a $70 \%$ alcohol scrub followed by placement of a chlorhexidineimpregnated disk over the catheter insertion site. A transparent polyurethane dressing (Bioclusive Transparent 